\title{
RESEARCH
}

Open Access

\section{Comparison of different methods for the estimation of aortic pulse wave velocity from 4D flow cardiovascular magnetic resonance}

Sophia Houriez--Gombaud-Saintonge ${ }^{1,2,3}$, Elie Mousseaux ${ }^{4}$, loannis Bargiotas ${ }^{5}$, Alain De Cesare ${ }^{1,3}$, Thomas Dietenbeck ${ }^{1,3}$, Kevin Bouaou ${ }^{1,3}$, Alban Redheuil ${ }^{1,3}$, Gilles Soulat ${ }^{4}$, Alain Giron ${ }^{1}$, Umit Gencer ${ }^{4}$, Damian Craiem ${ }^{6}$, Emmanuel Messas ${ }^{4}$, Emilie Bollache ${ }^{1,3}$, Yasmina Chenoune ${ }^{2 \dagger}$ and Nadjia Kachenoura ${ }^{1,3^{*}+}$

\begin{abstract}
Background: Arterial pulse wave velocity (PW) is associated with increased mortality in aging and disease. Several studies have shown the accuracy of applanation tonometry carotid-femoral PWV (Cf-PWV) and the relevance of evaluating central aorta stiffness using 2D cardiovascular magnetic resonance (CMR) to estimate PWV, and aortic distensibility-derived PWV through the theoretical Bramwell-Hill model (BH-PWV). Our aim was to compare various methods of aortic PWV (aoPWV) estimation from 4D flow CMR, in terms of associations with age, Cf-PWV, BH-PWV and left ventricular (LV) mass-to-volume ratio while evaluating inter-observer reproducibility and robustness to temporal resolution.
\end{abstract}

Methods: We studied 47 healthy subjects (49.5 \pm 18 years) who underwent Cf-PWV and CMR including aortic 4D flow CMR as well as 2D cine SSFP for BH-PWV and LV mass-to-volume ratio estimation. The aorta was semiautomatically segmented from 4D flow data, and mean velocity waveforms were estimated in 25 planes perpendicular to the aortic centerline. 4D flow CMR aoPWV was calculated: using velocity curves at two locations, namely ascending aorta (AAo) and distal descending aorta (DAo) aorta (S1, 2D-like strategy), or using all velocity curves along the entire aortic centreline (3D-like strategies) with iterative transit time (TT) estimates (S2) or a plane fitting of velocity curves systolic upslope (S3). For S1 and S2, $\pi$ was calculated using three approaches: crosscorrelation (TTC), wavelets (TTw) and Fourier transforms (TTf). Intra-class correlation coefficients (ICC) and BlandAltman biases (BA) were used to evaluate inter-observer reproducibility and effect of lower temporal resolution.

Results: 4D flow CMR aoPWV estimates were significantly $(p<0.05)$ correlated to the CMR-independent Cf-PWV, $\mathrm{BH}-\mathrm{PW}$, age and LV mass-to-volume ratio, with the strongest correlations for the 3D-like strategy using wavelets $T \mathrm{~T}$ (S2-TTW) $(R=0.62,0.65,0.77$ and 0.52 , respectively, all $p<0.001$ ). S2-TTw was also highly reproducible (ICC $=0.99$, $B A=0.09 \mathrm{~m} / \mathrm{s})$ and robust to lower temporal resolution $(I C C=0.97, B A=0.15 \mathrm{~m} / \mathrm{s})$.

Conclusions: Reproducible 4D flow CMR aoPWV estimates can be obtained using full 3D aortic coverage. Such 4D flow CMR stiffness measures were significantly associated with Cf-PW, BH-PWV, age and LV mass-to-volume ratio, with a slight superiority of the 3D strategy using wavelets transit time (S2-TTW).

Keywords: Pulse wave velocity, 4D flow CMR, Aortic stiffness, Aging

\footnotetext{
* Correspondence: nadjia.kachenoura@inserm.fr

${ }^{\dagger}$ Yasmina Chenoune and Nadjia Kachenoura contributed equally to this work.

'Sorbonne Université, INSERM, CNRS, Laboratoire d'Imagerie Biomédicale

(LIB), 75006 Paris, France

${ }^{3}$ Institute of Cardiometabolism and Nutrition (ICAN), Paris, France

Full list of author information is available at the end of the article
}

(c) The Author(s). 2019 Open Access This article is distributed under the terms of the Creative Commons Attribution 4.0 International License (http://creativecommons.org/licenses/by/4.0/), which permits unrestricted use, distribution, and reproduction in any medium, provided you give appropriate credit to the original author(s) and the source, provide a link to the Creative Commons license, and indicate if changes were made. The Creative Commons Public Domain Dedication waiver (http://creativecommons.org/publicdomain/zero/1.0/) applies to the data made available in this article, unless otherwise stated. 


\section{Background}

Aortic stiffening is an early sign of remodeling and functional changes in arterial hemodynamics, and a marker of cardiovascular aging $[1,2]$. The clinical usefulness of aortic stiffness has been previously demonstrated through its significant associations with adverse left ventricular (LV) remodeling, coronary heart disease, atherosclerosis and elevated mortality [3-6]. Aortic stiffness is commonly assessed using carotid-femoral (Cf) pulse wave velocity (PWV), which has been shown to be an accurate noninvasive alternative [7-9] to cardiac catheterization [10] in the in vivo measurement of pulse wave velocity (PWV). PWV is defined as the distance (D) travelled by the pressure wave between two anatomical locations, divided by the transit time (TT) spent by the wave to travel such distance.

Cardiovascular magnetic resonance imaging (CMR) offers excellent anatomical coverage and its anatomical and velocity-encoded sequences allow an accurate estimation of aortic geometry (length, diameters, volumes) as well as blood flow-derived indices in the thoracic aorta. In particular, two-dimensional through-plane phase-contrast CMR (2D phase contrast (PC)-CMR) has been used for the estimation of aortic (ao) PWV (aoPWV), using arch length from the ascending (AAo) to the descending (DAo) aorta, divided by the TT derived from a single acquisition plane positioned perpendicularly to both the AAo and DAo [11-14]. Alternatively, ascending aorta PWV estimation was also proposed using the theoretical BramwellHill (BH) model and aortic distensibility, which is commonly derived from aortic cine CMR and central pulse pressure [15-17].

CMR with full three-dimensional anatomical coverage and velocity encoding in the three directions resolved throughout the cardiac cycle (4D flow CMR) has been developed, opening new and unique opportunities to both visualize and quantify cardiovascular complex blood flow [18, 19]. 4D flow CMR has several advantages, including its excellent 3D anatomical and velocity coverage which enables an accurate estimation of aortic arch length and aortic flow rates and velocities. Furthermore, as compared with 2D PC-based approaches, 4D flow PWV is better suited to diseases with complex arterial geometry and tortuosity or with heterogeneous stiffness patterns along the arterial tree that can be associated with atherosclerosis [20] or changes in arterial size [21, 22]. Moreover, the estimation of aortic PWV from 4D flow CMR [20] has been shown to be feasible using either TT [20, 23-25] or plane fitting [20] approaches. The present study aims to provide a comprehensive comparison of both TT and plane fitting-based methods for aortic PWV estimation from 4D flow CMR in healthy subjects, in terms of: 1) associations with the CMR-independent well-established Cf-PWV measure,
4D flow CMR-independent BH-PWV, age and LV massto-volume ratio, as well as 2) inter-observer reproducibility and robustness to temporal resolution.

\section{Methods \\ Study population and data acquisition}

We retrospectively studied 47 healthy subjects 20 to 79 years ( $49.5 \pm 18$ years, 23 males), without overt cardiovascular disease. Approval of the local Institutional Review Board and informed consent was obtained from all participants. All subjects had a CMR exam on a $3 \mathrm{~T}$ system (Discovery 750w GEM, General Electric Healthcare, Waukesha, Wisconsin, USA), including 4D flow acquisitions with retrospective electrocardiogram (ECG) gating in a sagittal oblique volume encompassing the thoracic aorta. The three-directional encoding velocity was equal to $250 \mathrm{~cm} / \mathrm{s}$ and the scan parameters were as follows: flip angle $=15^{\circ}$, reconstructed voxel size $=1.5 \times 2.4 \times 1 \mathrm{~mm}^{3}$, echo time $=1.7 \mathrm{~ms}$, repetition time $=4.3$ to $4.4 \mathrm{~ms}$, acquisition matrix $=256 \times 96 \times 136$ and views per segment $=2$, resulting in acquired temporal resolution $=34.4$ to 35.2 ms, which was then reconstructed into 50 frames per cardiac cycle while applying a view sharing technique. Acquisition time was around $10 \mathrm{~min}$. Under-sampled k-space data were reconstructed using an algorithm combining iterative autocalibrating parallel imaging and compressed sensing with nonlinear $\ell 1$-norm wavelet regularization (L1 SPIR-iT) [26-28] with a factor of acceleration of 2 for the slice direction and 2.4 for the phase direction. 4D flow CMR datasets of 13 randomly selected subjects $(44 \pm 13$ years) were also reconstructed using 20 frames per cardiac cycle to study the effect of temporal resolution on aortic PWV estimation. Injection of $0.20 \mathrm{mmol} / \mathrm{kg}$ gadolinium contrast agent (gadobenate dimeglumine, Guerbet, France) was performed just prior to $4 \mathrm{D}$ flow CMR acquisition.

Blood pressures were measured using a Sphygmocor Xcel (AtCor Medical, Australia) device simultaneously to CMR acquisitions, and central systolic (SBP) and diastolic (DBP) blood pressures were recorded. Pulse pressure ( $\mathrm{PP}=$ SBP-DBP) was subsequently calculated. All subjects also underwent an applanation tonometry exam immediately after CMR acquisitions to compute the Cf-PWV, as previously described [29], to provide an CMR-independent arterial PWV measure.

In addition, cine balanced steady-state free precession (bSSFP) images were acquired during breath-holds perpendicular to the aorta at the level of pulmonary artery bifurcation to simultaneously image both AAo and DAo as well as in short axis views to cover the whole LV, using the following scan parameters: acquisition matrix = $260 \times 192$, repetition time $=3.7 \mathrm{~ms}$, echo time $=1.5 \mathrm{~ms}$, flip angle $=50^{\circ}$, pixel size $=0.74 \mathrm{~mm} \times 0.74 \mathrm{~mm}$, slice thickness $=8 \mathrm{~mm}$, views per segment $=12 . \mathrm{LV}$ data were 
analysed using Qmass 6 Software (Medis, Leiden, the Netherlands) while semi-automatically tracing endocardial and epicardial contours on all contiguous short axis slices, resulting in LV end-diastolic (EDV) and endsystolic (ESV) volumes as well as LV mass (LVM). The LV mass-to-volume ratio (LVM/EDV) was calculated and used as a measure of LV remodeling [30].

Aortic bSSFP images were analysed using the validated ArtFun software (Sorbonne Université/U.1146 Inserm) [31], which automatically delineated AAo lumen contours for all phases of the cardiac cycle. Then, local AAo BH-PWV was calculated from AAo distensibility (AAo systolic area-AAo diastolic area)/(AAo diastolic area $\cdot$ central PP) according to the Bramwell-Hill (BH) model [15-17] i.e. BH-PWV $=1 / \sqrt{\rho \times \text { distensibility }}$ where $\rho$ is blood density set to $1060 \mathrm{~kg} \cdot \mathrm{m}^{-3}$.

\section{Estimation of aortic pulse wave velocity from 4D flow CMR data}

Background phase offsets and phase wrapping were corrected as previously recommended [23]. Besides, to improve the quality of aortic segmentation, a 3D PC CMR angiogram was computed [32] while combining the modulus and velocity images averaged over time phases around the systolic peak, defined as the temporal phase with maximal velocity in the AAo. Aortic volume segmentation from the PC-CMR angiogram was then used to mask velocities through the cardiac cycle and to extract the aortic centerline and subsequently aortic length, using a semi-automated custom segmentation software (Mimosa, Sorbonne Université/Inserm 1146) [33]. Finally, 25 planes which were equally spaced and automatically positioned perpendicularly to the aortic centerline between the distal DAo and AAo, were used to calculate 25 mean velocity curves along the aorta which were interpolated with a time step of $1 \mathrm{~ms}$ using a spline function (Fig. 1a).

Three different strategies were then used for aoPWV estimation. While the first strategy (Strategy 1) was equivalent to a 2D PC-CMR strategy based on two anatomical locations, strategy 2 and 3 included all the 3D information.

\section{Strategy 1}

aoPWV was computed as the distance (D) along the centerline between two extreme planes located in AAo and distal DAo, divided by TT calculated from normalized mean velocity curves at these two locations: aoPWV = D/TT (Fig. 1b). Of note, methods used for TT estimation are described below. Contrary to a throughplane 2D PC-CMR PWV measurement, a more extended segment of the aorta was included to keep PWV Cramer-Rao lower error bound $\left(\sigma_{c}\right)$ reasonable, and thus to minimize the error on PWV estimation [12] while considering image characteristics such as temporal and spatial resolutions as well as signal to noise ratio.

$$
\sigma_{c} \geq \frac{c 2}{S N R \times \phi_{\max }} \sqrt{\frac{6 \times \Delta t \times \Delta x \times t_{\text {rise }}}{L^{3}}}
$$

$\sigma_{\mathrm{c}}$ :Cramer-Rao lower error bound; c: estimation of PWV; $\Delta \mathrm{t}, \Delta \mathrm{x}$ : temporal, spatial resolution, respectively; $t_{\text {rise: }}$ systolic upslope duration; SNR: signal to noise ratio; $\phi_{\max }$ : phase angle at the maximal velocity; $\mathrm{L}$ : vessel length.

\section{Strategy 2}

aoPWV estimation used the full 3D anatomical coverage [24, 34]. First, intermediate distances $\left(D_{\mathrm{i}}\right)$ along the aortic centreline were obtained while considering the distal DAo plane and each successive plane until proximal AAo was reached (Fig. 1a). Second, the corresponding paired normalized mean velocity waveforms were used for $\mathrm{TT}_{\mathrm{i}}$ estimation. Such intermediate distances and transit times were plotted as $\mathrm{TT}_{\mathrm{i}}$ according to $\mathrm{D}_{\mathrm{i}}$ and, aoPWV was equal to the slope of the linear regression plot (Fig. 1c). Of note, methods used for $\mathrm{TT}_{\mathrm{i}}$ estimation are described below.

\section{Strategy 3}

aoPWV was estimated using the full 3D anatomical coverage of the aorta as well, but required no TT calculation [20]. First, normalized mean velocity curves in aortic planes positioned between the proximal AAo and distal DAo were plotted according to time and distance along the aortic centreline (Fig. 1a and d). Then, aoPWV was calculated from the parameters of the plane used to fit the upslope of these curves, which was restricted between the 2nd and 8th decile of the systolic upslope.

\section{Transit time estimation}

For Strategy 1 and Strategy 2, three methods of TT estimation previously described in the literature [15, 25] were tested (Fig. 2). The first method (TTc) is based on cross-correlation which was shown to be reproducible, robust and reliable using 2D PC-CMR $[35,36]$. The two other methods are based on wavelet (TTw) and Fourier (TTf) transforms, respectively, and were shown to be robust to poor temporal resolution [37, 38]. For all methods, TT is the time delay between two mean velocity curves or their systolic upslope measured at two different locations. Systolic upslope was restricted between its 2nd and the 8th decile. Technical details regarding TT estimates, which are described below, are provided in Fig. 2. 


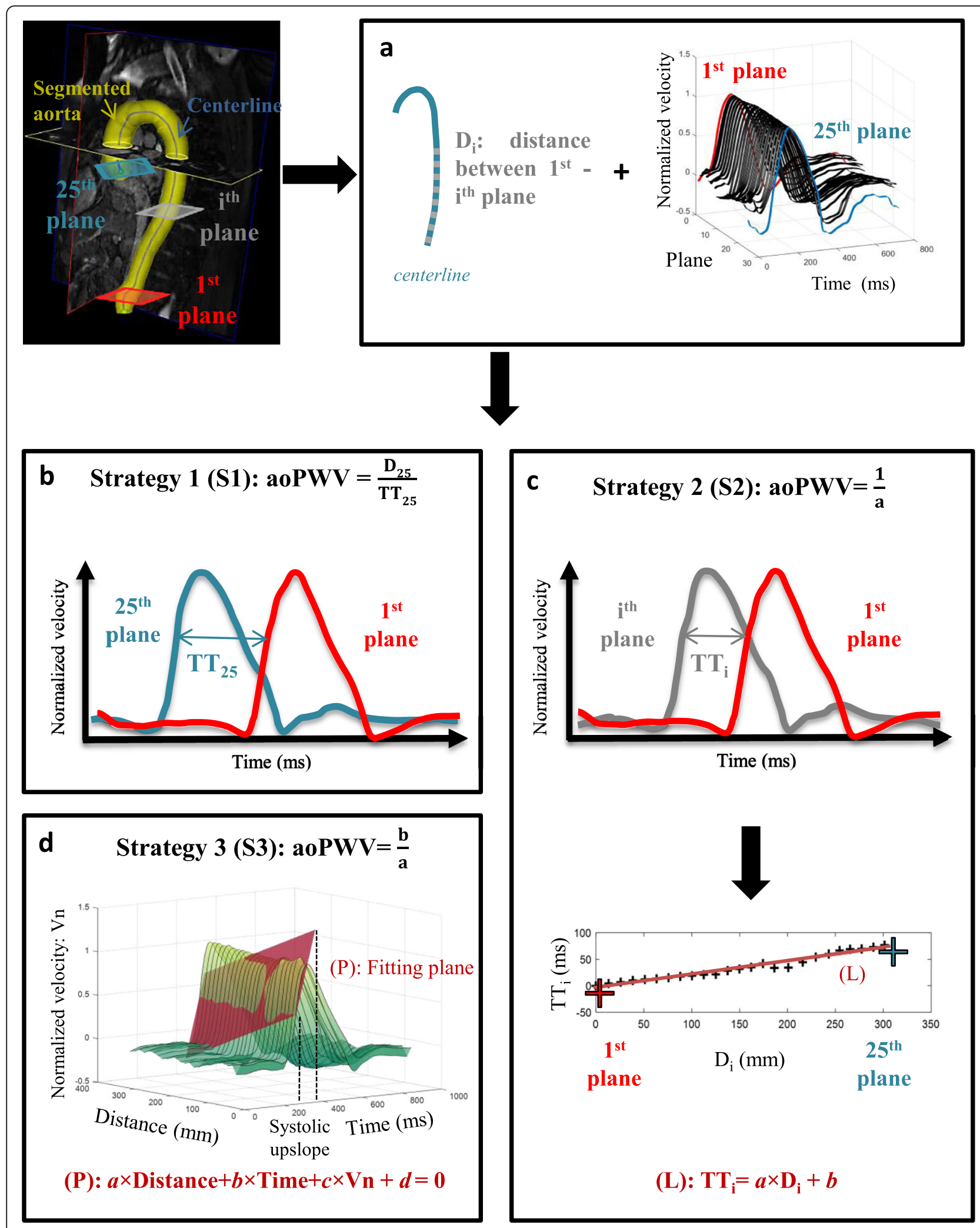

Fig. 1 (See legend on next page.) 
(See figure on previous page.)

Fig. 1 Aortic pulsed wave velocity (PWV) estimation from 4D flow CMR. a Shared pre-processing step for all aortic PWV (aoPWV) strategies: aortic 3D segmentation, positioning of cross-sectional planes perpendicular to the aortic centreline between the distal descending aorta (1st plane) and proximal ascending aorta (25th plane), extraction of the corresponding 25 mean velocity curves, as well as Di distances between the 1st and the ith planes along the centreline. Of note, mean velocity curves were interpolated with a 1-ms time step using a spline function. $\mathbf{b}$ For strategy 1 (S1), aoPWV was defined as the ratio of the distance $\left(\mathrm{D}_{25}\right)$ between the most distal (\#1) and most proximal (\#25) planes to the transit time $\left(T_{25}\right)$ estimated from the corresponding normalized velocity waveforms. Transit time estimation methods are described in Fig. 2. c For strategy 2 (S2), intermediate distances (Di) and transit times $\left(\mathrm{TT}_{\mathrm{i}}\right)$ were estimated as in $\mathrm{S} 1$ between plane 1 and each successive plane (\#i), until the proximal ascending aortic plane is reached. Such process provides the plot at the bottom, which was then linearly fitted $\left(L: T_{i}=a^{*} D_{i}+b\right)$. aoPWV was equal to reciprocal of the slope (a). d For strategy 3 (S3), transit time estimation was not needed since aoPWV was defined from the parameters of the plane $(P: a \times$ distance $+b \times$ time $+c \times$ velocity $+d=0)$ used to fit the systolic upslope of the normalized velocity curves corresponding to the 25 aortic planes illustrated in panel $\mathbf{a}$

\section{Cross-correlation transit time (TTC)}

TT was equal, in the time domain, to the time shift which maximizes the correlation between the two mean velocity curves [39]. The measurement of TTc (Fig. 2b) was performed on normalized mean velocity curves while considering only the systolic upslope [40, 41] to minimize the effects of backward flow, which occurs mostly in late systolic or diastolic phases.

\section{Wavelet transit time (TTw)}

TT estimation in the time-frequency domain was derived from the 4-order complex Gaussian wavelet transform, which was initially applied to the normalized mean velocity curves at two aortic locations [37]. The complex cross-spectrum phase difference and modulus derived from such wavelet transforms were then restricted to the systolic upslope, similarly to the crosscorrelation method. Finally, TTw was equal to the sum of the cross-spectrum phase difference weighted by its modulus (Fig. 2c) [37].

\section{Fourier transit time (TTf)}

TT estimation was modelled by the group delay of a filter which takes as input and output Fourier transforms $(\mathrm{X}(\mathrm{f})$ and $\mathrm{Y}(\mathrm{f}))$ of two normalized mean velocity curves $(x(t)$ and $y(t)$, respectively) measured at two aortic locations. Such filter transfer function was calculated as $\mathrm{H}(\mathrm{f})=\mathrm{Y}(\mathrm{f}) / \mathrm{X}(\mathrm{f})$. TTf was then equal to the group delay weighted by the harmonics of the input signal (Fig. 2d). In this frequency domain-based method [38], the entire time interval rather than systolic upslope was considered.

\section{Inter-observer reproducibility analysis}

The only source of variability was the aortic segmentation, while the remaining steps for 4D flow CMR aoPWV estimation were fully automated. Accordingly, such segmentation along with computation of aoPWV using the above-mentioned strategies were performed on a subgroup of 15 randomly selected subjects $(47 \pm 22$ years), in a random order by two independent operators (SHGS and KB with 2-year experience in 4D flow image processing), both blinded to subjects characteristics and to each other's segmentation and quantitative results.

\section{Statistical analysis}

Basic characteristics and PWV estimates are provided as median and interquartile range. The study population was divided into two subgroups according to age $(<$ or $\geq$ 50 years) and Wilcoxon rank-sum test was used for comparison between the two age subgroups. Univariate linear regression was performed to study the relationships of 4D flow CMR aoPWV estimates, with Cf-PWV, BHPWV, age, and LV mass-to-volume ratio. Regarding associations with LV mass-to-volume ratio, further multivariate models including potential confounders such as age, body mass index (BMI), SBP and gender were studied. Correlation coefficients $\mathrm{R}$ and estimated coefficient $\beta$ are provided. Bland-Altman analyses were performed for: 1) comparison between 4D flow CMR methods and Cf-PWV as well as BH-PWV, to provide the positioning of 4D flow CMR measures against well-established PWV measures, 2) studying 4D flow CMR aoPWV interoperator reproducibility and the effect of lower temporal resolution by comparing results of the 20 and 50 phases reconstructions, 3) comparison of the 4D flow CMR aoPWV method that was shown to be superior to the other methods based on the analyses described above, against the remaining 4D flow CMR aoPWV methods. For all Bland-Altman analyses, mean biases (MB), limits of agreements (LA) defined as mean bias $\pm 1.96 \times$ Standard deviation [42] were provided. Intraclass correlation coefficients (ICC) were also calculated. Finally, the Wilcoxon rank-sum test was used to test for statistical significance of the differences between aoPWV values obtained by the two observers or for the two reconstructions. A result was reported as significant if $p<0.05$. Statistical analyses were performed in Matlab (Mathworks, Natick, Massachusetts, USA).

\section{Results}

Table 1 summarizes subjects characteristics. As expected, SBP and PP were significantly higher for older subjects when compared to younger subjects. The effect 
a

\section{Transit time (TT) computation ?}

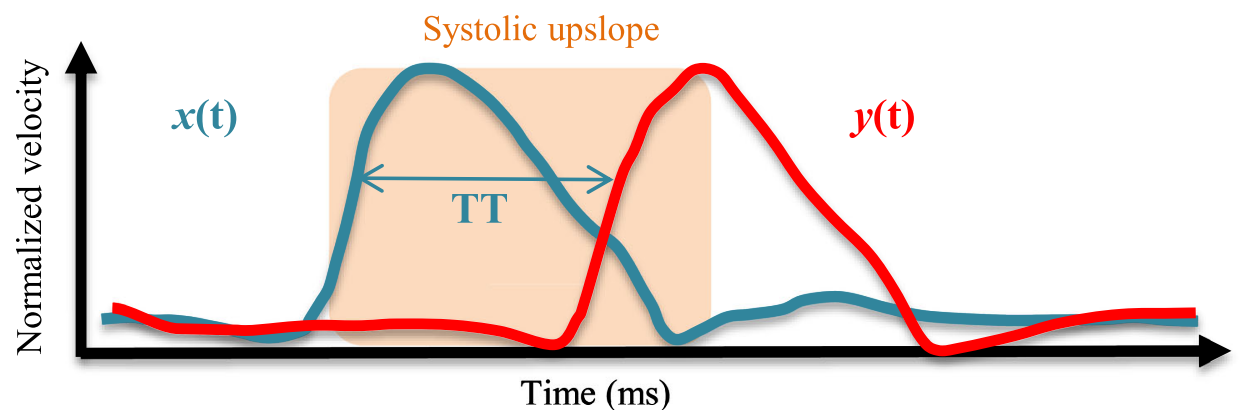

Time (ms)

\section{b Cross-Correlation (TTc)}

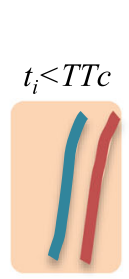

$\operatorname{Cor}\left(t_{i}\right)=\frac{\operatorname{Covariance}\left(\mathrm{x}\left(t+t_{i}\right), y(t)\right)}{\sqrt{\operatorname{Variance}\left(x\left(t+t_{i}\right)\right) \times \operatorname{Variance}(y(t))}}$ $0<t_{i}<\max ($ Time)

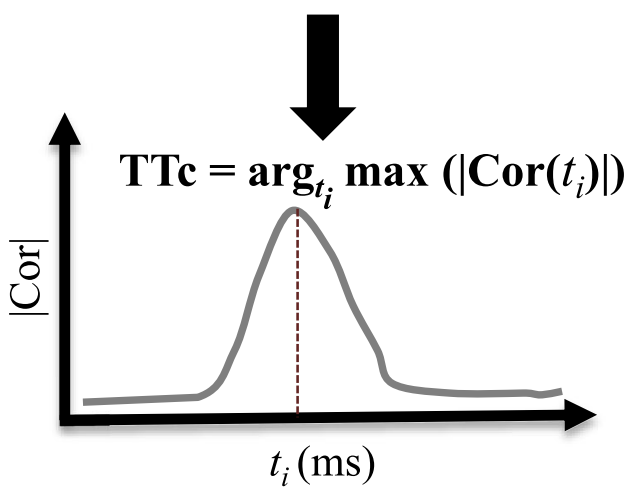

C Wavelet (TTw)

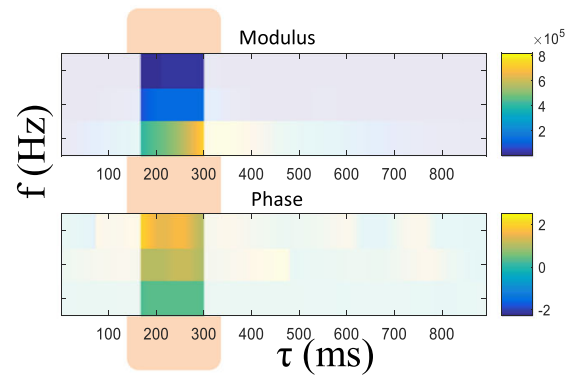

Cross Spectrum $W^{x y} \tau$

$$
\mathbf{T T w}=\sum_{\tau} \sum_{\mathbf{f}} \frac{\mathbf{W}_{\tau, \mathbf{f}}^{x y}}{\sum \mathbf{W}^{x y}{ }_{\tau, \mathbf{f}}} \times \frac{\varphi\left(\mathbf{W}^{x y}{ }_{\tau, \mathbf{f}}\right)}{2 \pi \times \mathbf{f}}
$$

\section{d Fourier (TTf)}

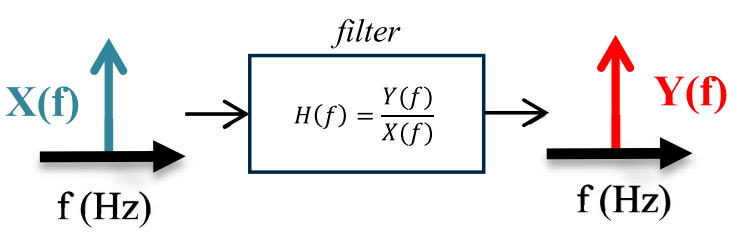

$$
\operatorname{TTf}=\frac{|\mathrm{X}|^{2} \times G D}{2 \pi \times \operatorname{sum}\left(|\mathrm{X}|^{2}\right)}
$$

With $G D=\frac{-d \varphi(H)}{d f}$ 
(See figure on previous page.)

Fig. 2 Transit time estimation from 4D flow CMR normalized time-resolved velocity curves. a Transit time (TT) is defined as the time shift needed to maximise the overlap between two normalized aortic velocity curves $x(t)$ and $y(t)$ extracted from two distinct planes positioned along the aortic centreline as illustrated in Fig. 1. Three TT estimation methods are used. $\mathbf{b}$ Cross-correlation transit-time (TT): a time shift ( $\mathrm{t}_{\mathrm{i}}$ ), comprised between 0 and the systolic duration is applied to $x(t)$ while maximizing its overlap with $y(t)$ in terms of systolic upslope. Such overlap was iteratively defined by the cross-correlation Cor $\left(\mathrm{t}_{\mathrm{i}}\right)$ between the shifted curve $x\left(t+t_{i}\right)$ and $y(t)$ systolic upslopes. TTc was set to the time shift maximizing the cross-correlation function Cor $\left(\mathrm{t}_{\mathrm{i}}\right)$. c Wavelet transform transit-time (TTw): 4th order Gaussian wavelet transform was applied on the normalized mean velocity curves $x(t)$ and $y(t)$ resulting in the provided modulus and phase of the cross-spectrum. TTw is then defined as the sum of such phase weighted by its modulus (see equation), while considering only the systolic upslope. $\mathbf{d}$ Fourier transform transit-time (TTf): time shift is modelled by the group delay introduced by a filter, which considers $x(t)$ as an input and $y(t)$ as an output. Normalized mean velocity curves $x(t)$ and $y(t)$ are Fourier transformed into $(X(f)$ and $Y(f))$ defining the filter transfer function $H(f)=Y(f) / X(f)$. TTf is calculated as the sum of the filter group delay (GD) weighted by the harmonics of the input signal (see equation)

of age was also observed on Cf-PWV, BH-PWV and LV mass-to-volume ratio (LVM/EDV), which were all significantly higher in the older subjects. All 4D flow CMR aoPWV values are also presented in Table 1 . They all showed a significant increase $(p<0.001)$ between the younger and older groups.

\section{Association between 4D flow CMR aoPWV and Cf-PWV as well as BH-PWV}

Correlation coefficients for comparisons of all 4D flowderived aoPWV estimates against reference tonometric Cf-PWV and ascending aorta BH-PWV are summarized in Table 2 and the corresponding linear regression plots are provided in Fig. 3. All 4D flow aoPWV estimates were significantly correlated $(p<0.05)$ with Cf-PWV as well as AAo BH-PWV, with the highest correlation coefficients obtained when using Strategy 2 along with the wavelet-based TT estimation (S2-TTw PWV: $R=0.62$, $p<0.001$ with Cf-PWV; $R=0.65, p<0.001$ with $\mathrm{BH}$ PWV) and Strategy 3 (S3 PWV: $R=0.61, p<0.001$ with Cf-PWV; $R=0.64, p<0.001$ with BH-PWV).

\section{Associations of 4D flow CMR aoPWV with age and left ventricular mass-to-volume ratio}

Correlation coefficients for associations of all 4D flow CMR aoPWV values with age and with LV mass-to-volume ratio are shown in Table 2. All 4D flow-derived aoPWV measures were significantly associated with age, with the

Table 1 Subjects characteristics and pulse wave velocity estimates

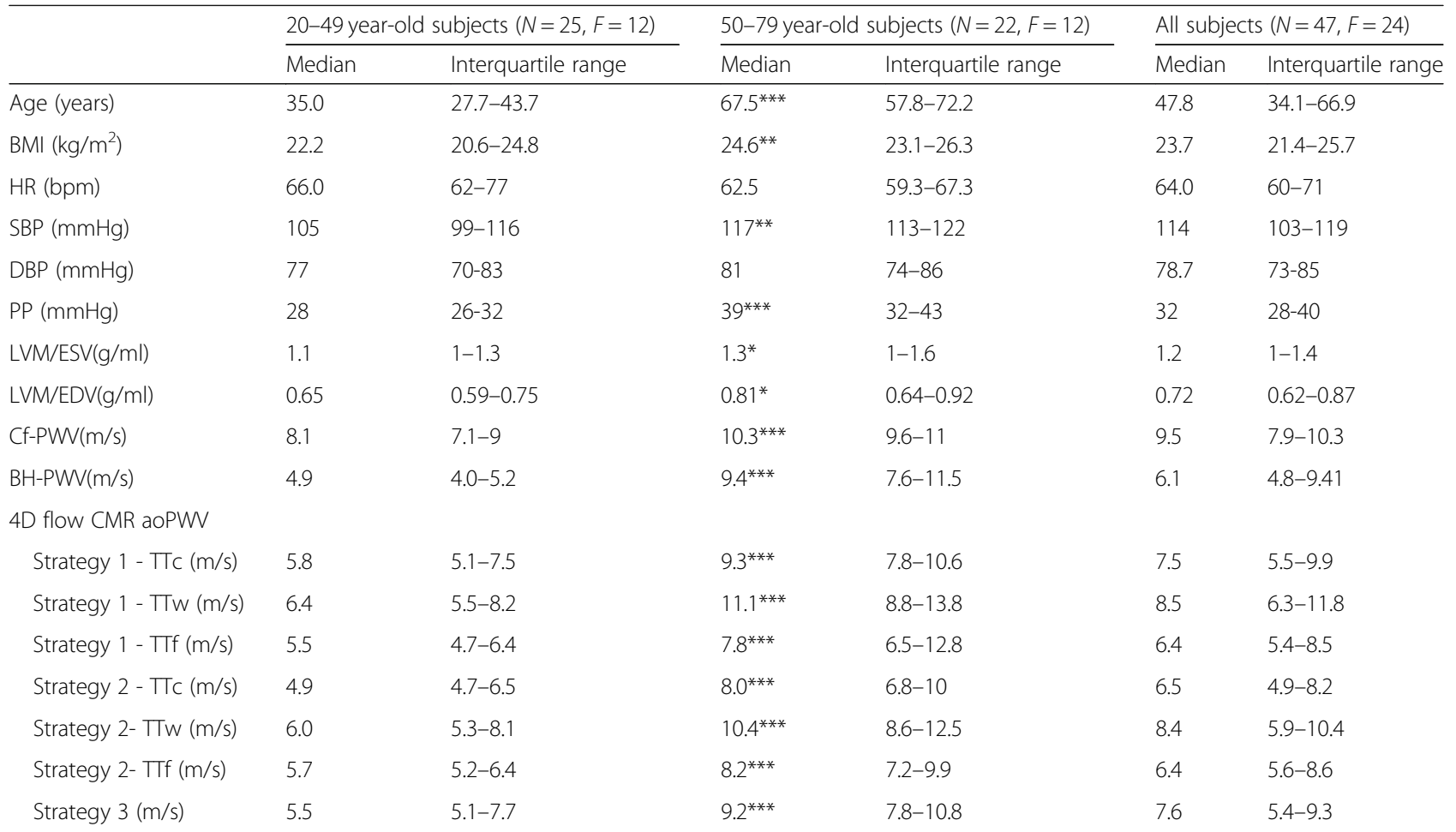

$N$ number of subjects, $F$ number of women, $B M I$ body mass index, $H R$ heart rate, $S B P$ systolic blood pressure, $D B P$ diastolic blood pressure, $P P$ pulse pressure, $C F-$ $P W V$ carotid-femoral pulse wave velocity, LVM/ESV and LVM/EDV left ventricular mass to end-systolic and end-diastolic volume ratios, $B H$-PWV ascending aorta pulse wave velocity according to the Bramwell Hill Model, $\Pi c, \Pi T w$ and $\Pi \pi f$ transit times estimated using cross-correlation, wavelets and Fourier transforms, respectively. Levels of significance were indicated by ${ }^{* *}$ for $p<0.001,{ }^{* *}$ for $p<0.01,{ }^{*}$ for $p<0.05$, for comparisons between the 2 age groups 
Table 2 Associations of 4D flow CMR aoPWV methods with CfPWV, BH-PWV, age and LV mass-to-volume ratio

\begin{tabular}{llllll}
\hline & & Cf-PWV & BH-PWV & Age & LV mass-to-volume ratio \\
\hline S1 & TTC & $0.30^{*}$ & $0.35^{*}$ & $0.44^{* *}$ & $0.39^{* *}$ \\
& TTW & $0.48^{* * *}$ & $0.56^{* * *}$ & $0.62^{* * *}$ & $0.44^{* *}$ \\
& TTf & $0.41^{* *}$ & $0.42^{* * *}$ & $0.39^{* *}$ & 0.14 \\
S2 & TTC & $0.55^{* * *}$ & $0.57^{* * *}$ & $0.66^{* * *}$ & $0.44^{* *}$ \\
& TTW & $0.62^{* * *}$ & $0.65^{* * *}$ & $0.77^{* * *}$ & $0.52^{* * *}$ \\
& TTf & $0.41^{* * *}$ & $0.44^{* * *}$ & $0.53^{* * *}$ & 0.18 \\
S3 & & $0.61^{* * *}$ & $0.64^{* * *}$ & $0.76^{* * *}$ & $0.47^{* * *}$ \\
\hline
\end{tabular}

Cf-PWV carotid-femoral (Cf) pulse wave velocity, $B H-P W V$ ascending aorta pulse wave velocity according to the Bramwell Hill Model, $\Pi c, \pi w$ and $T \pi f$ transit times estimated using cross-correlation, wavelets and Fourier transforms, respectively. Correlation coefficients are provided along with their levels of significance, which were indicated by ${ }^{* * *}$ for $p<0.001,{ }^{* *}$ for $p<0.01,{ }^{*}$ for $p<0.05$

highest correlations obtained for S2-TTw $(R=0.77$, $p<0.001)$ and S3 $(R=0.76, p<0.001)$. Of note, such correlations were in the same range as the correlations between age and reference Cf-PWV $(R=0.72$, $p<0.001)$ as well as BH-PWV $(R=0.79, p<0.001)$. Interestingly, association with age remained significant even in the elderly group for both S2-TTw $(R=0.59$, $p<0.001)$ and S3 $(R=0.59, p<0.001)$ aoPWV measures. Significant correlations with age in univariate analysis remained significant $(p<0.001)$ after adjustment for BMI, gender and SBP without other significant correlates.

For associations with LV mass-to-volume ratio, 4D flow CMR aoPWV obtained using S2-TTw $(R=0.52$, $p<0.001)$ and S3 $(R=0.47, p<0.001)$ resulted in the highest correlations in univariate analysis. Such correlations were even slightly higher than those obtained for the reference Cf-PWV $(R=0.39, p<0.01)$ and equivalent to those obtained for BH-PWV $(R=0.52, p<0.001)$. The associations with LV mass-to-volume ratio obtained for S2-TTw, S3, and Cf-PWV remained significant after adjustment for age, gender, BMI, SBP (Table 3). Of note, gender was also a significant correlate.

\section{Inter-observer reproducibility and effect of temporal resolution}

Results of inter-observer reproducibility are summarized in the left part of Table 4. For all methods, there were no significant differences between 4D flow CMR aoPWV values measured by the two operators $(p \geq 0.38)$. Bland-Altman mean biases and limits of agreement indicated a higher reproducibility of the 3D-based strategies (S2 and S3), as compared with the 2D-based strategy (S1).

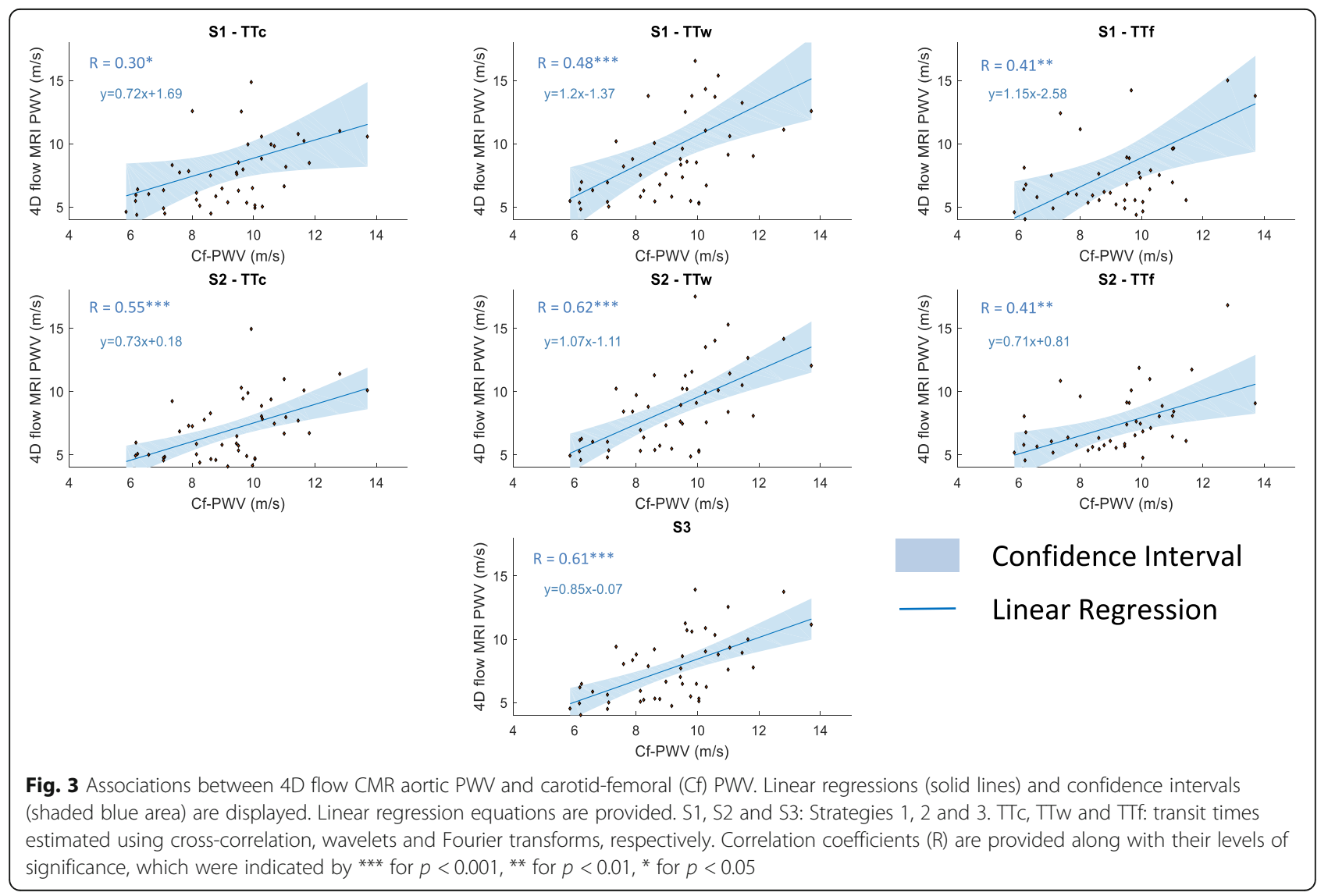


Table 3 Independent correlates of left ventricular mass-tovolume ratio

\begin{tabular}{|c|c|c|c|c|c|}
\hline & \multicolumn{2}{|c|}{ Model A } & \multicolumn{3}{|c|}{ Model B } \\
\hline & $\beta$ & $R$ & $\beta$ & $R$ & Significant correlates \\
\hline Cf-PWV & 0.038 & $0.39^{* *}$ & 0.037 & $0.667^{* * *}$ & Cf-PWV $^{*}$, Gender $^{* * *}$ \\
\hline S2-TTw & 0.028 & $0.52^{* * *}$ & 0.024 & $0.672^{* * *}$ & S2-TTw*, Gender ${ }^{* *}$ \\
\hline S3 & 0.032 & $0.47^{* * *}$ & 0.027 & $0.670^{* * *}$ & S3 ${ }^{*}$, Gender $^{* *}$ \\
\hline
\end{tabular}

Cf-PWV carotid-femoral pulse wave velocity, S2-TTW PWV estimated with Strategy 2 considering the, wavelets transform transit time, $\$ 3$ pulse wave velocity estimated with plane fitting. $\beta$ estimated coefficient, $R$ correlation coefficient. Model $A$ univariate regression of association between pulse wave velocity measures with LV mass-to-volume ratio (LVM/EDV). Model B Model $A+$ adjustment for age, gender (male), body mass index and systolic blood pressure. Levels of significance were indicated by ${ }^{* * *}$ for $p<0.001$, ** for $p<0.01,{ }^{*}$ for $p<0.05$

Analysis of the effect of reconstructed temporal resolution is summarized in the right part of Table 4, revealing that approaches using computation only in time domain (S1-TTc, S2-TTc, and S3) were less robust to the 20-phase reconstruction (ICC $<0.82$ ) than frequency (S1-TTf, S2-TTf) or time-frequency (S1-TTw, S2-TTw) domain ( $\mathrm{ICC}>0.91$ ) methods. Of note, at each given transit time method, the strategy with a 3D coverage performed better than the 2D plans strategy.

\section{Comparisons between the various PWV estimates}

Table 5 summarizes results of the Bland-Altman analyses for comparisons of the 4D flow CMR aoPWV methods with Cf-PWV and BH-PWV. The corresponding plots are shown and commented in the Additional file 1. For comparisons against Cf-PWV, mean bias was close to zero or negative, in line with higher stiffness of peripheral arteries as compared with central elastic arteries. For comparisons against ascending aortic $\mathrm{BH}-$ PWV, mean bias was close to zero or positive, highlighting the highest elasticity of the most proximal aortic segment. Comparison between the two 4D flow CMR aoPWV methods (S2-TTw and S3) that were shown to
Table 5 Bland-Altman analyses for comparisons of the 4D flow CMR aoPWV methods with Cf-PWV and BH-PWV

\begin{tabular}{|c|c|c|c|c|c|}
\hline & & \multicolumn{2}{|l|}{ Cf-PWV } & \multicolumn{2}{|l|}{ BH-PWV } \\
\hline & & Mean bias $(\mathrm{m} / \mathrm{s})$ & $\mathrm{LA}(\mathrm{m} / \mathrm{s})$ & Mean bias $(\mathrm{m} / \mathrm{s})$ & $\mathrm{LA}(\mathrm{m} / \mathrm{s})$ \\
\hline \multirow[t]{3}{*}{ S1 } & $\pi \mathrm{TC}$ & -0.9 & {$[-9.1 ; 7.3]$} & 0.9 & {$[-8.3 ; 10.1]$} \\
\hline & $\pi w$ & 0.5 & {$[-7.3 ; 8.3]$} & 2.2 & {$[-5.6 ; 10]$} \\
\hline & TTf & -1.2 & {$[-10.4 ; 8.0]$} & 0.5 & {$[-9.3 ; 10.3]$} \\
\hline \multirow[t]{3}{*}{ S2 } & Tाc & -2.3 & {$[-6.4 ; 1.8]$} & -0.5 & {$[-6.8 ; 5.8]$} \\
\hline & $\pi w$ & -0.5 & {$[-5.4 ; 4.4]$} & 1.2 & {$[-4.9 ; 7.3]$} \\
\hline & TTf & -1.8 & {$[-7.4 ; 3.8]$} & -0.1 & {$[-7.5 ; 7.3]$} \\
\hline S3 & & -1.4 & {$[-5.3 ; 2.5]$} & 0.3 & {$[-5.6 ; 6.2]$} \\
\hline
\end{tabular}

Bland-Altman mean biases and limits of agreement (LA) for comparisons between 4D flow MRI aoPWV values and carotid-femoral PWV (Cf-PWV) as well as the ascending aorta Bramwell-Hill PWV (BH-PWV). Bias was defined as 4D flow MRI aoPWV - Cf-PWV or BH-PWV. S1, S2 and S3: Strategies 1, 2 and 3, respectively. $\Pi \mathrm{c}, \Pi \mathrm{T}$ and $\Pi \mathrm{Tf}$ : transit times estimated using cross-correlation, wavelets and Fourier transforms, respectively

be superior to other estimates, in terms of reproducibility as well as associations with Cf-PWV, BH-PWV, age, and LV mass-to-volume ratio, resulted in the highest ICC and the narrowest limits of agreement (Fig. 4).

\section{Discussion}

This study provides a comparison of the main methods available in the literature for aortic PWV estimation, using 4D flow CMR in 47 healthy subjects. The significant associations with age and the non-invasive reference applanation tonometry-derived Cf-PWV $(p<0.001)$ demonstrated the consistency of all 4D flow CMR-derived aoPWV estimates. Methods taking into account the whole 3D aortic spatial coverage, specifically the method based on the wavelet transform for transit time estimation were found to be superior to the other approaches, as revealed by 1) their stronger associations with Cf-PWV, Bramwell-Hill modelderived AAo PWV, age, and LV-mass-to-volume ratio as well as 2) their higher reproducibility and robustness to lower reconstructed temporal resolution. Interestingly,

Table 4 Inter-observer reproducibility and effect of 20- vs. 50-time reconstructed frames temporal resolution

\begin{tabular}{|c|c|c|c|c|c|c|c|c|c|}
\hline & & \multicolumn{4}{|c|}{ Inter-observer reproducibility } & \multicolumn{4}{|c|}{ Effect of temporal resolution } \\
\hline & & Mean bias (m/s) & $\mathrm{LA}(\mathrm{m} / \mathrm{s})$ & $\mathrm{p}$ & ICC & Mean bias (m/s) & $L A(m / s)$ & $p$ & ICC \\
\hline \multirow[t]{3}{*}{ S1 } & $\pi \mathrm{TC}$ & -0.50 & {$[-2.6 ; 1.6]$} & 0.59 & 0.95 & -1.62 & {$[-11 ; 8.1]$} & 0.49 & 0.47 \\
\hline & $\pi w$ & 0.46 & {$[-5.2 ; 6.1]$} & 1.00 & 0.86 & 0.29 & {$[-1 ; 1.5]$} & 0.50 & 0.91 \\
\hline & $\pi f$ & -0.46 & {$[-3.4 ; 2.4]$} & 0.43 & 0.91 & 0.35 & {$[-0.5 ; 1.2]$} & 0.50 & 0.93 \\
\hline \multirow[t]{3}{*}{ S2 } & $\pi c$ & 0.06 & {$[-0.6 ; 0.7]$} & 0.84 & 0.99 & -0.96 & {$[-5.5 ; 3.6]$} & 0.54 & 0.75 \\
\hline & $\pi w$ & 0.09 & {$[-0.7 ; 0.8]$} & 0.80 & 0.99 & 0.15 & {$[-0.5 ; 0.8]$} & 0.76 & 0.97 \\
\hline & TTf & -0.53 & {$[-4.2 ; 3.2]$} & 0.38 & 0.61 & 0.15 & {$[-0.6 ; 0.9]$} & 0.92 & 0.97 \\
\hline S3 & & 0.18 & {$[-0.9 ; 1.2]$} & 0.84 & 0.97 & 0.33 & {$[-1.4 ; 2]$} & 0.68 & 0.82 \\
\hline
\end{tabular}

Measures of repeatability of 4D flow MRI aoPWV estimates are provided: Bland-Altman mean biases and limits of agreement (LA) as well as intra class correlation coefficients (ICC). $p$ values correspond to Wilcoxon rank-sum test of comparisons between aoPWV values obtained by the two observers and for the two reconstructions. S1, S2 and S3: Strategies 1, 2 and 3. TTc, TTw and TTf: transit times estimated using cross-correlation, wavelets and Fourier transforms, respectively 


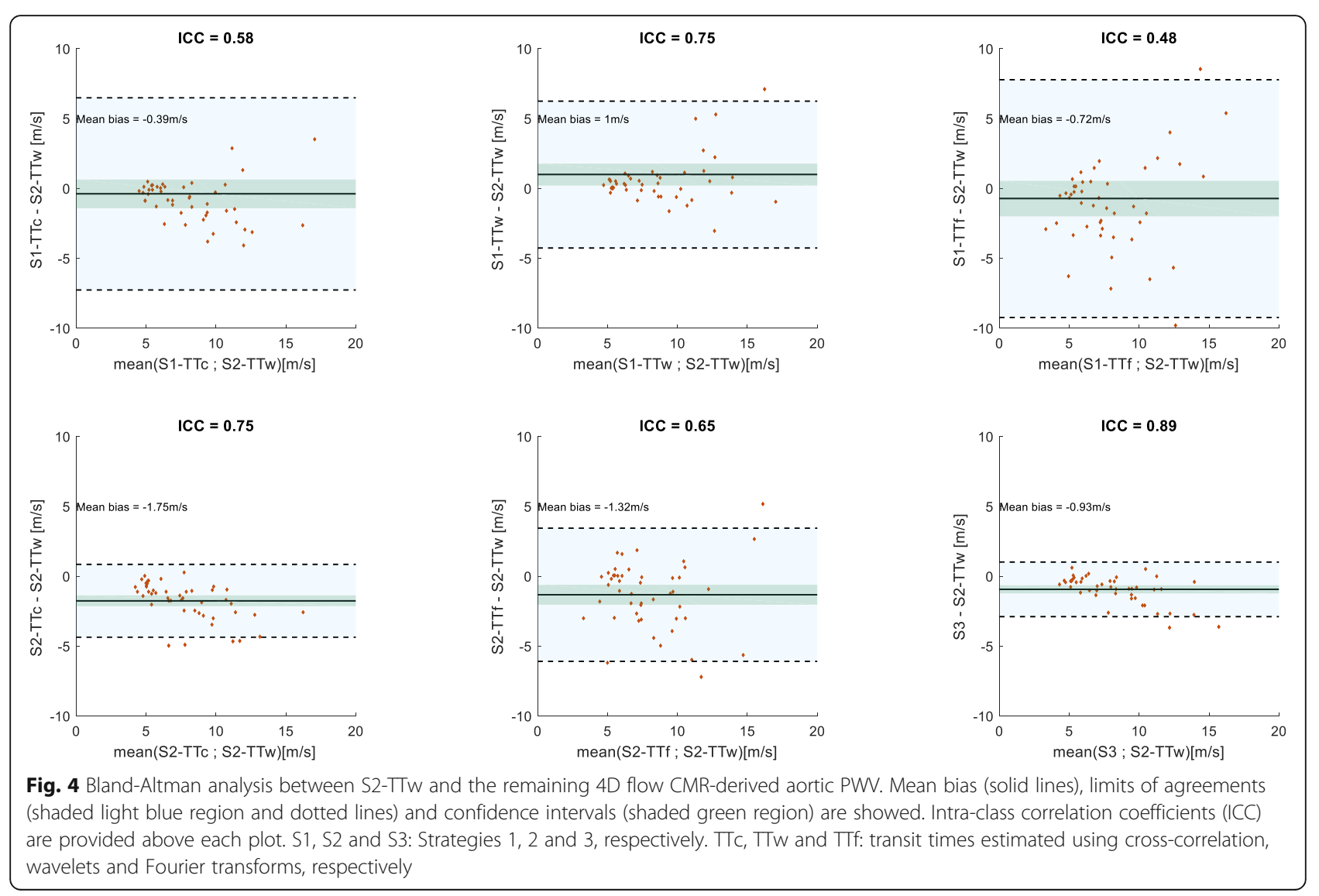

correlations of 4D flow CMR aoPWV methods, which account for the full 3D coverage, with age and LV mass-to-volume ratio were in the same range or even slightly higher than those obtained when using CfPWV, and remained significant after adjustment for the main confounders.

Aortic PWV values obtained in our study from 4D flow data are in a similar range using all methods. Furthermore, comparison against the widely available 2D PC-CMR aortic arch PWV values estimated in large populations in the literature revealed no substantial differences. Indeed, normal values for aortic arch PWV summarized by KawelBoehm et al. [43] were $3.9 \pm 1.1 \mathrm{~m} / \mathrm{s}$ for age range 30-39 years, $5.6 \pm 1.4 \mathrm{~m} / \mathrm{s}$ for $40-49$ years, $7.2 \pm 2.3 \mathrm{~m} / \mathrm{s}$ for 50 59 years, $9.7 \pm 2.9 \mathrm{~m} / \mathrm{s}$ for $60-69$ years, $11.1 \pm 4.6 \mathrm{~m} / \mathrm{s}$ for age $\geq 70$ years. Normal values for $4 \mathrm{D}$ flow CMR-derived aoPWV are not available yet since the majority of previous studies was performed on small groups or pathological individuals while using various methods. Nevertheless, despite differences in population age, our values were in the same range or only slightly higher than those provided in a recent $4 \mathrm{D}$ flow study [44] while using similar TT-based approaches $(n=8$, age $=23 \pm 2$ years: $\mathrm{PWV}=5.7 \pm 0.7 \mathrm{~m} / \mathrm{s}$ when using Fourier analysis and PWV $=5.5 \pm 0.7 \mathrm{~m} / \mathrm{s}$ when using cross-correlation; $n=8$, age $=58 \pm 2$ years:
PWV $=9.3 \pm 1.3 \mathrm{~m} / \mathrm{s}$ when using Fourier analysis and $\mathrm{PWV}=8.9 \pm 1.4 \mathrm{~m} / \mathrm{s}$ when using cross-correlation).

Our choice of the tested methods for aoPWV estimation in this study was based on previous 2D PC-CMR and 4D flow CMR studies. Indeed they have demonstrated that: 1) approaches based on a single point of the flow curve (foot or peak) are hampered by low velocity to noise ratio or by the effects of wave reflection $[24,36,45], 2)$ frequency and time-frequency domain methods for TT estimation are more robust to low temporal resolution [37, 38], and 3) approaches taking into account volumetric flow data along the aorta are more robust than methods considering only two measurement sites [20,24]. The high reproducibility of PWV previously shown in 2D PC-CMR studies was not found in our 2D-like strategy (S1), which resulted in sizeable Bland-Altman limits of agreements in our data. Such lower performances of the 2D-like strategies in our study might be explained by the static nature of the 4D flow CMR aortic lumen segmentation through time, contrary to an automated dynamic time-resolved segmentation in 2D PC-CMR, which would hamper the AAo velocity curves, as well as by the lower spatial resolution of $4 \mathrm{D}$ flow as compared to 2D PC-CMR data, which would highly affect the distal descending aorta because of its small cross-section. 
In agreement with previous findings [36, 37], systolic upslope-based methods were more consistent than wave-based methods especially when using the 3D strategy, as revealed by the comparison between wavelet or cross-correlation and Fourier approaches in terms of associations with age and LV mass to volume ratio. This can be explained by the fact that the early systolic upslope is less distorted by wave reflexion than late systolic and diastolic phases of the flow curve $[35,36]$. Besides, methods considering the volumetric coverage of 4D flow CMR data were more reliable than those based on two planes only.

Original features of our study include semi-automated segmentation of the aorta and an automated positioning of flow measurement planes perpendicular to the centreline. In addition, comparisons against the CMR-independent tonometry Cf-PWV and 4D flow CMR-independent ascending aortic BH-PWV measures, as well as the assessment of physiological associations with LV mass-to-volume ratio and age were reported. Our aoPWV measurements confirmed physiological knowledge on arterial stiffening gradient from the central aorta to peripheral arteries, resulting in the lowest values for the ascending aortic BramwellHill method, intermediate values for the 4D flow CMR methods over the whole aorta, and the highest values for the carotid to femoral arteries PWV. This stiffness gradient phenomenon was more marked in younger subjects with highly elastic central arteries than in elderly subjects, because of stiffness homogenisation from central arteries towards the periphery with ageing.

Furthermore, comparison to Cf-PWV and BH-PWV revealed the superiority of the plane fitting method as well as wavelet TT-based approach previously shown using 2D-PC CMR to be more robust to low temporal resolution [37]. Our findings further confirm such robustness of the wavelet-based method to 20- vs. 50reconstructed time frames. In addition, such method was strongly associated with age even in subjects $\geq 50$ years, in whom we expect increased aortic stiffness and thus decreased TT when compared to younger subjects. Although the wavelet-based approach resulted in the highest performances when compared to the plane fitting approach, its implementation requires tuning of parameters such as the mother wavelet and sampling frequency. However, one might highlight that the same parameters than those previously described using 2D-PC CMR data [37] were used in each subject for the wavelet method in the present study.

Reliability of our 4D flow CMR aoPWV measurement was also demonstrated by the positive association with LV mass-to-volume ratio. Indeed, it is known that in the process of healthy aging, arterial stiffening is associated with increased LV afterload and subsequent LV hypertrophic remodeling, which was measured in our study through the LV mass to end-diastolic volume ratio [30]. Such association was independent of age, systolic blood pressure, BMI, and gender, revealing that $4 \mathrm{D}$ flow CMR aoPWV is an independent marker of LV alteration in the process of healthy aging. Gender was also an independent correlate of LV mass-to-volume ratio, in agreement with a previous study [46]. Accordingly, evaluation of aortic stiffness using 4D flow CMR can provide mechanistic knowledge to improve understanding of ventricular-arterial coupling.

The main limitation of our study is the lack of a direct aortic PWV gold standard measurement as provided by catheterization [10]. However such invasive procedure was not feasible in healthy subjects. Moreover, associations with the non-invasive reference Cf-PWV [1, 7, 29], and with physiological criteria such as age and LV massto-volume ratio were used to compare the performances of 4D flow CMR aoPWV methods. Since 4D flow CMR is limited by low spatial resolution and signal to noise ratio for low velocities, time-resolved segmentation was not available. Accordingly, a fixed segmentation on an enhanced aortic angiogram, reconstructed from peak systolic phases, was used along with mean velocity curves rather than flow curves. In the future, a time-resolved segmentation would be necessary for an accurate estimation of aortic flow along the cardiac cycle. Such segmentation would require an evolvement of 4D flow CMR sequences with the use of higher spatial resolution and multiple encoding velocities to improve velocity to noise ratio especially in regions with low velocities or the use of motioncompensated compressed-sensing techniques which were shown to improve the quality of $4 \mathrm{D}$ flow images $[47,48]$. To enhance contrast and signal to noise ratio, contrast agent is often used in 4D flow CMR. However, since standard CMR exams in clinical routine usually include LGE and/or post-contrast T1, 4D flow CMR acquisition can be interleaved while waiting for such tissue characterization sequences to be acquired. Another limitation is the lack of test-retest variability assessment of the 4D flow CMR aoPWV due to non-available data. However, one might highlight that the main goal of our study was to isolate the technical reliability of $4 \mathrm{D}$ flow CMR aoPWV from the robustness of 4D flow CMR to hemodynamic changes over time. Thus, the proposed $4 \mathrm{D}$ flow CMR techniques were rather compared in terms of inter-observer reproducibility and associations with $4 \mathrm{D}$ flow CMR-independent aoPWV measures such as the tonometric Cf-PWV and the cine bSSFP-derived $\mathrm{BH}$ PWV as well as physiological association with age and LV mass-to-volume ratio.

\section{Conclusion}

Aortic PWV estimation in healthy subjects from 4D flow CMR data while considering the entire volumetric 
coverage of the aorta and a time-frequency wavelet approach for transit time calculation was reproducible, robust to lower reconstructed temporal resolution and reliable, as revealed by the strong associations with the well-established reference Cf-PWV, BH-PWV and physiological criteria such as age and LV mass-to-volume ratio. The LV and the aorta being simultaneously acquired in the same hemodynamic conditions when using 4D flow CMR, the derived aortic PWV might help for a better understanding of LV - aortic coupling.

\section{Supplementary information}

Supplementary information accompanies this paper at https://doi.org/10. 1186/s12968-019-0584-X.

Additional file 1. Bland-Altman plots for comparisons of $4 D$ flow MRI aoPWV with Cf-PWV and BH-PWV.

\section{Abbreviations}

AAo: Ascending aorta; aoPWV: Aortic pulse wave velocity; BH: Bramwell-Hill; BMI: Body mass index; bSSFP: Balanced steady-state free precession; Cf: Carotid-femoral; CMR: Cardiovascular magnetic resonance imaging; D: Distance; DAo: Descending aorta; DBP: Diastolic blood pressure; ECG: Electrocardiogram; EDV: End-diastolic volume; ESV: End-systolic volume; ICC: Intra-class correlation coefficient; LV: Left ventricle/left ventricular; LVM: Left ventricular mass; PC: Phase-contrast; PP: Pulse pressure; PWV: Pulse wave velocity; SBP: Systolic blood pressure; TT: Transit time

\section{Acknowledgments}

We would like to thank Dr. Marcus Alley, Senior Research Scientist in the Department of Radiology and Radiological Sciences Laboratory of the Stanford University School of Medicine, for his input regarding the technical details of the $4 \mathrm{D}$ flow MRI acqusition sequence

\section{Authors' contributions}

All authors contributed significantly to this manuscript. All authors: manuscript drafting or manuscript revision for important intellectual content. All authors: approval of final version of submitted manuscript. All authors: literature research. SH, EM, GS, UG, AG, EMe, EB YC, NK study concepts/study design or data acquisition or data and statistical analysis or findings interpretation. SH, TD, AD, KB, IB, NK, software design for quantification and analysis. YC and NK contributed equally to this work.

\section{Funding}

We acknowledge the FRM project ING20150532487 for funding Kevin Bouaou and ESME-Sudria for funding Sophia Houriez--Gombaud-Saintonge as well as the ECOS-SUD project number A15S04 (France-Argentina) exchange grant for funding a fruitful exchange around $4 D$ flow CMR data processing.

\section{Availability of data and materials}

The datasets used and/or analysed during the current study are available from the corresponding author on reasonable request.

\section{Ethics approval and consent to participate}

This study conformed to local ethics committee regulations. Ethics committee approval was waived for this study type. Signed informed consent was given by all participants.

\section{Consent for publication}

Not applicable.

\section{Competing interests}

The authors declare that they have no competing interests

\section{Author details}

'Sorbonne Université, INSERM, CNRS, Laboratoire d'Imagerie Biomédicale (LIB), 75006 Paris, France. ${ }^{2}$ ESME Sudria Research Lab, Paris, France. ${ }^{3}$ Institute of Cardiometabolism and Nutrition (ICAN), Paris, France. ${ }^{4}$ Hopital Européen Georges Pompidou, Paris, France. ${ }^{5}$ CMLA, ENS Cachan, CNRS, Université Paris-Saclay, 94235 Cachan, France. ${ }^{6}$ Universidad Favaloro-CONICET, IMeTTyB, Buenos Aires, Argentina.

Received: 12 November 2018 Accepted: 22 October 2019

Published online: 12 December 2019

\section{References}

1. Laurent S, Boutouyrie P, Asmar R, Gautier I, Laloux B, Guize L, et al. Aortic stiffness is an independent predictor of all-cause and cardiovascular mortality in hypertensive patients. Hypertension. 2001;37:1236-41.

2. Safar ME, London GM. Therapeutic studies and arterial stiffness in hypertension: recommendations of the European Society of Hypertension. The clinical Committee of Arterial Structure and Function. Working group on vascular structure and function of the European Society of Hypertension. J Hypertens. 2000;18:1527-35.

3. Meaume S, Benetos A, Henry OF, Rudnichi A, Safar ME. Aortic pulse wave velocity predicts cardiovascular mortality in subjects $>70$ years of age. Arterioscler Thromb Vasc Biol. 2001;21:2046-50.

4. Ben-Shlomo Y, Spears M, Boustred C, May M, Anderson SG, Benjamin EJ, et al. Aortic pulse wave velocity improves cardiovascular event prediction: an individual participant meta-analysis of prospective observational data from 17,635 subjects. J Am Coll Cardiol. 2014;63:636-46.

5. Sutton-Tyrrell K, Najjar SS, Boudreau RM, Venkitachalam L, Kupelian V, Simonsick EM, et al. Elevated aortic pulse wave velocity, a marker of arterial stiffness, predicts cardiovascular events in well-functioning older adults. Circulation. 2005;111:3384-90.

6. Willum-Hansen T, Staessen JA, Torp-Pedersen C, Rasmussen S, Thijs L, Ibsen $\mathrm{H}$, et al. Prognostic value of aortic pulse wave velocity as index of arterial stiffness in the general population. Circulation. 2006;113:664-70.

7. Collaboration TRV for AS. Determinants of pulse wave velocity in healthy people and in the presence of cardiovascular risk factors: establishing normal and reference values. Eur Heart J. 2010;31:2338-50.

8. Alecu C, Labat C, Kearney-Schwartz A, Fay R, Salvi P, Joly L, et al. Reference values of aortic pulse wave velocity in the elderly. J Hypertens. 2008;26: 2207-12.

9. Van Bortel LM, Balkestein EJ, van der Heijden-Spek JJ, Vanmolkot FH, Staessen JA, Kragten JA, et al. Non-invasive assessment of local arterial pulse pressure: comparison of applanation tonometry and echo-tracking. J Hypertens. 2001;19:1037-44.

10. Latham RD, Westerhof N, Sipkema P, Rubal BJ, Reuderink P, Murgo JP. Regional wave travel and reflections along the human aorta: a study with six simultaneous micromanometric pressures. Circulation. 1985;72:1257-69.

11. Grotenhuis HB, Westenberg JJM, Steendijk P, van der Geest RJ, Ottenkamp J, Bax JJ, et al. Validation and reproducibility of aortic pulse wave velocity as assessed with velocity-encoded MRI. J Magn Reson Imaging. 2009:30:521-6.

12. Bolster BD, Atalar E, Hardy CJ, McVeigh ER. Accuracy of arterial pulse-wave velocity measurement using MR. J Magn Reson Imaging. 1998;8:878-88.

13. Hickson SS, Butlin M, Graves M, Taviani V, Avolio AP, McEniery CM, et al. The relationship of age with regional aortic stiffness and diameter. JACC Cardiovasc Imaging. 2010;3:1247-55.

14. Vulliémoz S, Stergiopulos N, Meuli R. Estimation of local aortic elastic properties with MRI. Magn Reson Med. 2002;47:649-54.

15. Dogui A, Kachenoura N, Frouin F, Lefort M, De Cesare A, Mousseaux E, et al. Consistency of aortic distensibility and pulse wave velocity estimates with respect to the Bramwell-Hill theoretical model: a cardiovascular magnetic resonance study. J Cardiovasc Magn Reson. 2011;13:11.

16. Westenberg JJ, van Poelgeest EP, Steendijk P, Grotenhuis HB, Jukema J, de Roos A. Bramwell-Hill modeling for local aortic pulse wave velocity estimation: a validation study with velocity-encoded cardiovascular magnetic resonance and invasive pressure assessment. J Cardiovasc Magn Reson. 2012;14:2.

17. Bramwell JC, Hill AV. The velocity of the pulse wave in man. Proc R Soc Lond B Biol Sci. 1922;93:298-306.

18. Stankovic Z, Allen BD, Garcia J, Jarvis KB, Markl M. $4 D$ flow imaging with MRI. Cardiovasc Diagn Ther. 2014;4:173-92. 
19. Markl M, Schnell S, Barker AJ. 4D flow imaging: current status to future clinical applications. Curr Cardiol Rep. 2014;16:481.

20. Markl M, Wallis W, Strecker C, Gladstone BP, Vach W, Harloff A. Analysis of pulse wave velocity in the thoracic aorta by flow-sensitive four-dimensional MRI: reproducibility and correlation with characteristics in patients with aortic atherosclerosis. J Magn Reson Imaging. 2012;35:1162-8.

21. Guala A, Camporeale C, Ridolfi L, Mesin L. Non-invasive aortic systolic pressure and pulse wave velocity estimation in a primary care setting: an in silico study. Med Eng Phys. 2017;42:91-8.

22. Harloff A, Mirzaee H, Lodemann T, Hagenlocher P, Wehrum T, Stuplich J, et al. Determination of aortic stiffness using 4D flow cardiovascular magnetic resonance - a population-based study. J Cardiovasc Magn Reson. 2018;20:43.

23. Dyverfeldt $P$, Bissell M, Barker AJ, Bolger AF, Carlhäll C-J, Ebbers T, et al. 4D flow cardiovascular magnetic resonance consensus statement. J Cardiovasc Magn Reson. 2015;17:72

24. Markl M, Wallis W, Brendecke S, Simon J, Frydrychowicz A, Harloff A. Estimation of global aortic pulse wave velocity by flow-sensitive 4D MRI. Magn Reson Med. 2010;63:1575-82.

25. Wentland AL, Wieben O, François CJ, Boncyk C, Rio AMD, Johnson KM, et al. Aortic pulse wave velocity measurements with Undersampled 4D flowsensitive MRI: comparison to 2D and algorithm determination. J Magn Reson Imaging. 2013;37:853-9.

26. Vasanawala SS, Alley MT, Hargreaves BA, Barth RA, Pauly JM, Lustig M. Improved pediatric MR imaging with compressed sensing. Radiology. 2010;256:607-16.

27. Lustig M, Pauly JM. SPIRiT: iterative self-consistent parallel imaging reconstruction from arbitrary k-space. Magn Reson Med. 2010;64:457-71.

28. Vasanawala S, Murphy M, Alley M, Lai P, Keutzer K, Pauly J, et al. Practical parallel imaging compressed sensing MRI: summary of two years of experience in accelerating body MRI of pediatric patients. Proc IEEE Int Symp Biomed Imaging. 2011;2011:1039-43. https://doi.org/10.1109/ISBI. 2011.5872579

29. Laurent S, Cockcroft J, Van Bortel L, Boutouyrie P, Giannattasio C, Hayoz D, et al. Expert consensus document on arterial stiffness: methodological issues and clinical applications. Eur Heart J. 2006;27:2588-605.

30. Cheng S, Fernandes VRS, Bluemke DA, McClelland RL, Kronmal RA, Lima JAC. Age-related left ventricular remodeling and associated risk for cardiovascular outcomes: the multi-ethnic study of atherosclerosis. Circ Cardiovasc Imaging. 2009;2:191-8.

31. Herment A, Kachenoura N, Lefort M, Bensalah M, Dogui A, Frouin F, et al. Automated segmentation of the aorta from phase contrast MR images: validation against expert tracing in healthy volunteers and in patients with a dilated aorta. J Magn Reson Imaging. 2010;31:881-8.

32. Markl M, Harloff A, Bley TA, Zaitsev M, Jung B, Weigang E, et al. Timeresolved $3 D$ MR velocity mapping at $3 \mathrm{~T}$ : improved navigator-gated assessment of vascular anatomy and blood flow. J Magn Reson Imaging. 2007;25:824-31.

33. Dietenbeck T, Craiem D, Rosenbaum D, Giron A, De Cesare A, Bouaou K et al. 3D aortic morphology and stiffness in MRI using semi-automated cylindrical active surface provides optimized description of the vascular effects of aging and hypertension. Comput Biol Med. 2018;103:101-8.

34. Spottiswoode B, Stalder AF, Gulsun MA, Campione KF, Carr M, Wasielewski $M$, et al. Fast semi-automated analysis of pulse wave velocity in the thoracic aorta using high temporal resolution 4D flow MRI. J Cardiovasc Magn Reson. 2013;15:P87.

35. Ibrahim E-SH, Johnson KR, Miller AB, Shaffer JM, White RD. Measuring aortic pulse wave velocity using high-field cardiovascular magnetic resonance: comparison of techniques. J Cardiovasc Magn Reson. 2010;12:26.

36. Dogui A, Redheuil A, Lefort M, DeCesare A, Kachenoura N, Herment A, et al. Measurement of aortic arch pulse wave velocity in cardiovascular MR: comparison of transit time estimators and description of a new approach. J Magn Reson Imaging. 2011;33:1321-9.

37. Bargiotas I, Mousseaux E, Yu W-C, Venkatesh BA, Bollache E, de Cesare A et al. Estimation of aortic pulse wave transit time in cardiovascular magnetic resonance using complex wavelet cross-spectrum analysis. J Cardiovasc Magn Reson. 2015;17:65.

38. Meloni A, Zymeski H, Pepe A, Lombardi M, Wood JC. Robust estimation of pulse wave transit time using group delay. J Magn Reson Imaging. 2014;39:550-8.

39. Ou P, Celermajer DS, Raisky O, Jolivet O, Buyens F, Herment A, et al. Angular (gothic) aortic arch leads to enhanced systolic wave reflection, central aortic stiffness, and increased left ventricular mass late after aortic coarctation repair: evaluation with magnetic resonance flow mapping. J Thorac Cardiovasc Surg. 2008;135:62-8.

40. Westerhof BE, Guelen I, Westerhof N, Karemaker JM, Avolio A. Quantification of wave reflection in the human aorta from pressure alone. Hypertension. 2006:48:595-601.

41. Bensalah MZ, Bollache E, Kachenoura N, Giron A, Cesare AD, Macron L, et al. Geometry is a major determinant of flow reversal in proximal aorta. Am J Physiol - Heart Circ Physiol. 2014;306:H1408-16.

42. Bland JM, Altman D. Statistical methods for assessing agreement between two methods of clinical measurement. Lancet. 1986;327:307-10.

43. Kawel-Boehm N, Maceira A, Valsangiacomo-Buechel ER, Vogel-Claussen J, Turkbey EB, Williams R, et al. Normal values for cardiovascular magnetic resonance in adults and children. J Cardiovasc Magn Reson. 2015;17:29.

44. Dyverfeldt P, Ebbers T, Länne T. Pulse wave velocity with 4D flow MRl: systematic differences and age-related regional vascular stiffness. Magn Reson Imaging. 2014;32:1266-71.

45. Fielden SW, Fornwalt BK, Jerosch-Herold M, Eisner RL, Stillman AE, Oshinski $\mathrm{JN}$. A new method for the determination of aortic pulse wave velocity using cross-correlation on 2D PCMR velocity data. J Magn Reson Imaging. 2008;27:1382-7.

46. Douglas PS, Katz SE, Weinberg EO, Chen MH, Bishop SP, Lorell BH. Hypertrophic remodeling: gender differences in the early response to left ventricular pressure overload. J Am Coll Cardiol. 1998;32:1118-25.

47. Cheng JY, Hanneman K, Zhang T, Alley MT, Lai P, Tamir Jl, et al. Comprehensive motion-compensated highly accelerated 4D flow MRI with ferumoxytol enhancement for pediatric congenital heart disease. J Magn Reson Imaging. 2016:43:1355-68.

48. Cheng JY, Zhang T, Ruangwattanapaisarn N, Alley MT, Uecker M, Pauly JM, et al. Free-breathing pediatric MRI with nonrigid motion correction and acceleration. J Magn Reson Imaging. 2015;42:407-20.

\section{Publisher's Note}

Springer Nature remains neutral with regard to jurisdictional claims in published maps and institutional affiliations.
Ready to submit your research? Choose BMC and benefit from:

- fast, convenient online submission

- thorough peer review by experienced researchers in your field

- rapid publication on acceptance

- support for research data, including large and complex data types

- gold Open Access which fosters wider collaboration and increased citations

- maximum visibility for your research: over $100 \mathrm{M}$ website views per year

At $\mathrm{BMC}$, research is always in progress.

Learn more biomedcentral.com/submissions 